\title{
Excited-state properties of 20-chloro-chlorophyll a
}

\author{
D. Leupold ${ }^{\text {a }}$, A. Struck ${ }^{b}$, H. Stiel ${ }^{a}$, K. Teuchner ${ }^{a}$, S. Oberländer ${ }^{c}$ and H. Scheer ${ }^{b}$ \\ a Zentralinstitut für Optik und Spektroskopie, Akademie der Wissenschaften der DDR, \\ DDR-1199 Berlin-Adlershoj, German Democratic Republic \\ b Botanisches Institut der Universität München, D-8000 Munich 19, Federal Republic of Germany \\ c Karl-Weierstrass-Institut für Mathematik, Akademie der Wissenschaften der DDR, \\ DDR-1040 Berlin, German Democratic Republic
}

Received 29 December 1989; in final form 6 April 1990

The excited-state and lasing properties of 20-chloro-chlorophyll a in ether solution were compared to those of chlorophyll a. Desactivation parameters and cross-sections were obtained from non-linear absorption spectroscopy in combination with a physico-mathematical methods package. The $\mathrm{Cl}$ substituent at $\mathrm{C}$-20 (1) increases both intersystem crossing and internal conversion, (2) produces a blue-shift of the $S_{1}$ absorption spectrum, and (3) leads to pronounced photochemistry.

\section{Introduction}

Chlorophyll a (Chl a) chlorinated at the C-20 position has found interest during recent years, at first as a possible component of photosystem I of oxygenevolving organisms [1-3] (which is now ruled out [4-6]). More recently it has been related to the (biological?) degradation of chlorophylls [7]. Illumination of 20-chloro-chlorophyll a (20-Cl-Chl a) in acetonc solution with white light yielded a photoproduct with red-shifted $S_{0}-S_{1}$ absorption, which is transformed in the dark to a new species, probably a linear tetrapyrrole [7].

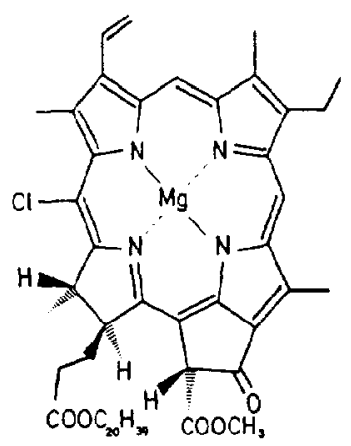

To characterize further the photo-chemistry and physics of 20-Cl-Chl a we have now investigated its first excited electronic state by laser spectroscopic methods. In the following, the excited-state absorption, fluorescence and lasing action of 20-Cl-Chl a are described and discussed in comparison with the unsubstituted Chl a. These studies have been performed in ether solution in order to characterize the excited-state properties under conditions where no photochemistry [7] takes place $\#$.

\section{Materials and methods}

$\mathrm{Chl} \mathbf{a}$ and 20-Cl-Chl a were prepared as in ref. [7]. The excited-state properties were determined by using the hardware and software of a "PhysicoMathematical Methods Package of Non-linear Absorption and Emission of Organic Molecules" [811]. In the first step of its general procedure, experimentally accessible functions and quantities are measured which implicitly contain the desired information. These are non-linear absorption, non-linear emission, time-resolved fluorescence and/or differential optical-density $(\Delta \mathrm{OD})$ spectra.

\#1 In acetone solution of $20-\mathrm{Cl}-\mathrm{Chl}$ a, we have found in the meantime further photochemical reactions, which will be the subject of a separate publication. 
In the second part, this implicit information is transformed into explicit statements, concerning, e.g., the minimum range and the structure of the molecular energy-level system involved in the non-linear light-matter interaction, and boundaries for unknown excited-state constants (e.g. absorption crosssections and relaxation constants). This is based on analytical investigations of the coupled system of rate equations for the population densities and the photon-transport equation(s) for the incoherent interaction (of generalized energy-level schemes) of organic molecules with laser pulses (partly described in ref. [12]). These analytical procedures are available in form of an expert system, realized as an interactive program for IBM PC/AT compatible computers.

This information was used as input for the numerical-treatment part of the package, which is also based on the aforementioned differential equation system and realized as microcomputer software. A starting set of the nonlinear functions is calculated and compared with the experimental curves. Then, using a weighted least-squares minimization method, the still unknown excited-state parameters are varied until the deviation of the calculated curve from the corresponding experimentally determined curve becomes smaller than the relative error of measurement. Special care is taken for the uniqueness of the optimum set of excited-state constants obtained in this way, both within the scope of the chosen model and with respect to the model itself (e.g. sensitivity analysis, variation of pulse duration in non-linear measurements [13], lasing properties, cf. below). With this set of constants, the quantitative excitedstate spectra can also be calculated from the $\Delta O D$ measurements.

For the application of this general procedure to the problems of the present work, the following three branches of the measuring device were used:

(i) Fluorescence spectroscopy module. Fluorescence spectra were measured with a modified laserpulse fluorometer LIF 200 (Academy of Sciences of the GDR) and quantum proportionally corrected by means of a calibrated tungsten lamp. Fluorescence quantum yields were determined by using $\mathrm{Chl} \mathbf{a}$ in ethanol as a reference $(\Phi=0.30[14])$. The solutions were excited at $337 \mathrm{~nm}(O D=0.1)$ and the quantum proportionally corrected spectra were in- tegrated taking into account the refractive index of the solvent. For fluorescence-lifetime measurements the LIS 202 laser-pulse spectrometer (JENAOPTIK, Jena) [15] was used. The low-temperature measurements were carried out by means of a special quartz dewar which allows fluorescence measurements of small spheres of the solution within liquid nitrogen. To do this, drops of the solution were given into the liquid nitrogen where glassy spheres were formed even for the solvent ether.

(ii) A non-linear absorption module [16]. The main parts are a nitrogen-laser-pumped tunable dye laser: $(\Delta t(\mathrm{fwhm})=1.7 \mathrm{~ns}, \Delta \lambda=0.1 \mathrm{~nm})$, a light attenuator working continuously over 5 orders of magnitude (maximum photon flux density $6 \times 10^{26} \mathrm{~cm}^{-2}$ $\mathrm{s}^{-1}$, measured at the sample position), and photodiode detection of the transmitted and reference light beams.

A signal analyzer MFA 105 (Academy of Sciences of the GDR ) processed the signals and controlled the measurement. The signals of both the reference and the measurement channel are accumulated separately. At the end of a measurement, the sum of transmission values at each intensity channel is divided by the corresponding number of laser pulses. With a sample completely insensitive to laser-induced absorption changes the relative error of the transmission measurement is about $1 \%$.

(iii) A transient spectroscopy module. This is based on a spectrograph with a CCD row, coupled with an optical multichannel analyzer (OVA 284, Academy of Sciences of the GDR). Both the excitation and probing pulses were realized by a nitrogen-laser- $(0.5 \mathrm{~ns} / 350 \mathrm{~kW} / 5 \mathrm{~Hz}$ repetition rate) pumped grazing-incidence dye laser; its monochromatic pulse $(\Delta \lambda=0.04 \mathrm{~nm})$ served for excitation and the spectrally broad ASE pulse $(\Lambda \lambda(f w h m) \geqslant 25 \mathrm{~nm})$ was used for probing (no delay), in some cases also for excitation. All transient spectra were accumulated over 100 pulses, each pulse was directed on a formerly non-illuminated part of the cell. This was realized by a pin-hole diaphragm and a low repetition-rate $(5 \mathrm{~Hz})$ laser regime coupled with a step motor-controlled motion of the cell perpendicular to the light-pulse direction.

The computerized data handling to obtain the differential optical-density spectra includes base-line 
corrections and reference signal-based calibration.

\section{Results}

\subsection{Fluorescence}

Fluorescence spectra of dilute ether solutions $\left(10^{-5}\right.$ M) of 20-Cl-Chl a and - for comparison - of Chl a were excited by low intensity nanosecond dye laser pulses and found to be independent of excitation wavelength. From these spectra the emission crosssections were calculated according to ref. [17] (fig. 1). To calibrate the latter the specific absorption coefficient of $\mathrm{Chl}$ a in ether was taken from ref. [18]. For 20-Cl-Chl a, the mean value according to five careful weighings was used. The fluorescence decay times and fluorescence quantum yields of both solutions at room temperature are shown in table 1. Both decays were found to be single exponential.

Also shown in table 1 are the resulting rate constants of radiative transition. For $\mathrm{Chl}$ a, a $10 \%$ contribution of internal conversion (IC) to $S_{1}$ deactivation was assumed according to ref. [14]. To

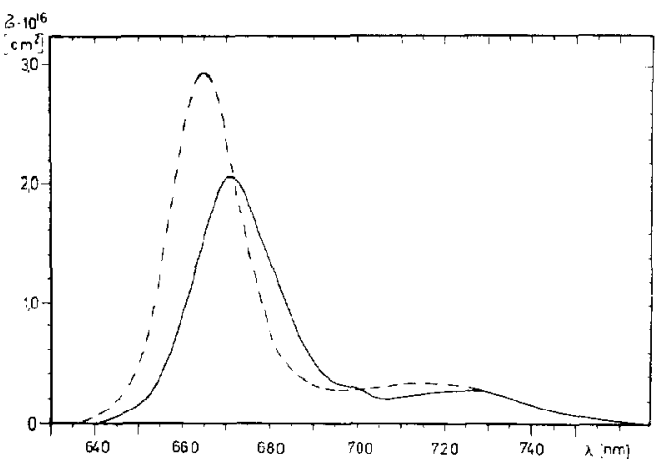

Fig. 1. Emission cross-sections of ether solutions of Chl a (--) and $20-\mathrm{Cl}-\mathrm{Chl}$ a $(-)$.

Table 1

Excited-state deactivation channels of $\mathrm{Chl} a$ and $20-\mathrm{Cl}-\mathrm{Chl} \mathrm{a}$ in diethyl ether

\begin{tabular}{llllll}
\hline & $\begin{array}{l}\tau_{\mathrm{F}} \\
(\mathrm{ns})\end{array}$ & $\Phi_{\mathrm{F}}$ & $\begin{array}{l}k_{\mathrm{rad}} \\
\left(\mathrm{s}^{-1}\right)\end{array}$ & $\begin{array}{l}k_{\mathrm{JC}} \\
\left(\mathrm{s}^{-1}\right)\end{array}$ & $\begin{array}{l}k_{\mathrm{ISC}} \\
\left(\mathrm{s}^{-1}\right)\end{array}$ \\
\hline 20-Cl-Chl a & 2.0 & 0.08 & $4.0 \times 10^{7}$ & $2.7 \times 10^{8}$ & $1.9 \times 10^{8}$ \\
Chl a & 6.0 & 0.32 & $5.3 \times 10^{7}$ & $1.7 \times 10^{7}$ & $1.0 \times 10^{8}$ \\
\hline
\end{tabular}

discriminate between the rates of intersystem crossing (ISC) $\left(k_{\mathrm{ISC}}\right)$ and IC $\left(k_{\mathrm{IC}}\right)$ for 20-Cl-Chl a, the fluorescence decay time at $77 \mathrm{~K}$ was measured ( $\tau=4.4 \mathrm{~ns}$ ). Assuming that $k_{\mathrm{ISC}}$ is temperature independent [19], we attributed the lengthening exclusively to the temperature dependence of the IC channcl (with $k_{\mathrm{IC}}=0$ at $77 \mathrm{~K}$ ), to yield the values given in table 1 . The assumption of zero IC contribution at $77 \mathrm{~K}$ was suggested by a corresponding $\tau_{\mathrm{F}}$ lengthening with $\mathrm{Chl} a$ at $77 \mathrm{~K}$.

\subsection{Non-linear absorption}

Non-linear absorption (intensity-dependent energy transmission) was measured for both solutions near the $\mathrm{S}_{0}-\mathrm{S}_{1}$ absorption maximum (sample thickness $0.1 \mathrm{~cm}$, low signal transmission $T_{0}=0.31(0.25)$ for $20-\mathrm{Cl}-\mathrm{Chl}$ a ( $\mathrm{Chl}$ a)). The experimental results for the two pigments in ether solution are shown in fig. 2 together with the level scheme used for simulation. Of the ten parameters (rate constants, $k$, and cross-sections, $\sigma$ ) per level scheme (table 2 ), the four labeled with asterisks are known as discussed above. $k_{51}$ was assumed at $10^{6}$; in view of the short-pulse excitation the exact value is of no relevance here. To get first approximations for some of the unknown parameters, the expert system was applied to the initial range of non-linear absorption. In this range, all

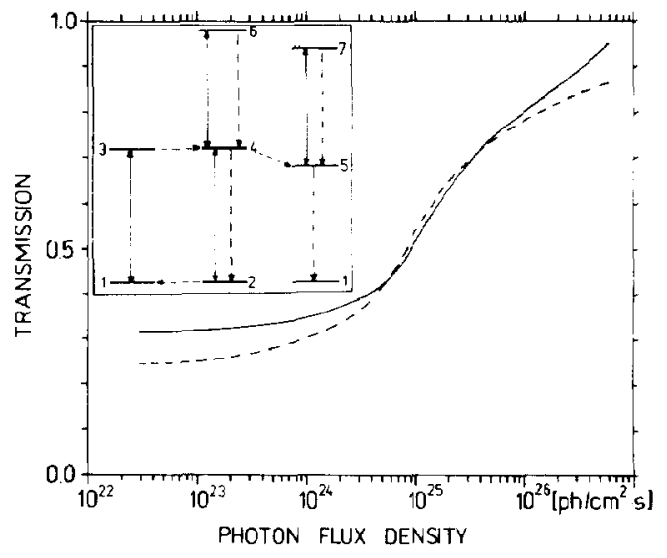

Fig. 2. Non-linear absorption of ether solutions of Chl a (---), measured at $\lambda=662 \mathrm{~nm}$ and of $20-\mathrm{Cl}-\mathrm{Chl}$ a $(-)$, measured at $\lambda=668 \mathrm{~nm}$. Also shown is the energy-level scheme used for simulation, with the following notations: (1) $S_{0}$, (2) $S_{0}$ (FC), (3) $S_{1}(F C),(4) S_{1},(5) T_{1},(6) S_{x}$, (7) $T_{x}$. 
Table 2

Parameter sets ( $a$ in $10^{-6} \mathrm{~cm}^{-2}, k$ in s$)^{-1}$ ) for simulation of the non-linear absorption curves of ether solutions of 20-Cl-Chl a ( $\lambda=668$ $\mathrm{nm})$ and $\mathrm{Chl} \mathrm{a}(\lambda=662 \mathrm{~nm})$, respectively, in diethylether shown in fig. 2 . The level scheme and notations are given in the inset of fig. 2

\begin{tabular}{|c|c|c|c|c|c|c|}
\hline & & ${ }^{*} \sigma_{13}, \sigma_{31}$ & ${ }^{*} \sigma_{42}, \sigma_{24}$ & $\sigma_{46}, \sigma_{64}$ & $\sigma_{57}, \sigma_{75}$ & \\
\hline & \multirow{2}{*}{$\begin{array}{l}20-\mathrm{Cl}-\mathrm{Chl} \mathrm{a} \\
\mathrm{Chl} \mathrm{a}\end{array}$} & \multirow{2}{*}{$\begin{array}{l}2.52 \\
3.80\end{array}$} & \multirow{2}{*}{$\begin{array}{l}2.06 \\
2.68\end{array}$} & \multirow{2}{*}{$\begin{array}{l}0.20 \\
1.00\end{array}$} & \multirow{2}{*}{\multicolumn{2}{|c|}{$\begin{array}{l}0.50 \\
0.50\end{array}$}} \\
\hline & & & & & & \\
\hline & $k_{21}, k_{34}$ & ${ }^{*} k_{42}$ & $k_{s 1}$ & ${ }^{*} k_{45}$ & $k_{61}$ & $k_{75}$ \\
\hline $20-\mathrm{Cl}-\mathrm{Chl}$ a & $10^{12}$ & $3.1 \times 10^{8}$ & $10^{6}$ & $1.9 \times 10^{8}$ & $10^{12}$ & $10^{12}$ \\
\hline Chl a & $10^{12}$ & $7.0 \times 10^{7}$ & $10^{6}$ & $1.0 \times 10^{8}$ & $5 \times 10^{11}$ & $10^{12}$ \\
\hline
\end{tabular}

processes starting at or below the terminal level of the first absorption transition come into play. In the present case, these are the Franck-Condon state relaxations $k_{21}$ and $k_{34}$, and the excited singlet and triplet absorptions $\sigma_{46}$ and $\sigma_{57}$ (see inset fig. 2 for terminology).

The expert system gives necessary relations between these parameters, which are used to select the starting set for the numerics. Sensitivity lests show that the non-linear functions are especially sensitive to $\sigma_{46}, \sigma_{57}$ and $k_{64}$. Therefore the minimization problem was solved for these three parameters, having fixed the others at values consistent with the mentioned analytical relations. The final results for both samples are shown in table 2 . The numerical simulation of non-linear absorption on the basis of these sets has a mean deviation from the measured curve of $2.7 \%(4.2 \%)$ in case of $\mathrm{Chl} \mathrm{a}(20-\mathrm{Cl}-\mathrm{Chl} \mathrm{a})$.

As shown below, the $\triangle O D$ measurements and lasing properties serve as a sensitive test of the first excited-singlet cross-section $\sigma_{46}$ and (because of the strong coupling) of $\sigma_{57}$ and $k_{64}$.

Based on the determined excited-state cross-sections and relaxation constants, the population density distribution for each useful excitation (within the limits of the model considered) can be calculated with the numerical package, e.g. as a basis of quantitative excited-state absorption spectra (vide infra). Based on these sets, it is also possible to calculate the excitation conditions for optimal selective excitation of any desired excited state [20].

\section{3. $S_{1}$ absorption within the $S_{0}-S_{1}$ absorption region}

For the determination of the $S_{1}$ absorption spectra, differential optical-density spectra $(\Delta O D)$ were measured using the short-pulse transient spectroscopy module described above. The excitation was located at $662 \mathrm{~nm}$ (Chl a) and $668 \mathrm{~nm}$ (20-Cl-Chl a), and emission continua from stilbene and DCM-dye solution (Lambda Physics) were used as probe pulses ( fwhm $=0.5 \mathrm{~ns}$ ).

The $S_{1}$ absorption cross-sections were calculated from these spectra by a special sub-program of the numerics package using the $S_{1}$ absorption and deactivation parameters obtained above. In these experiments the mean $S_{1}$ population during probing was $10 \%$ of the total chlorophyll concentration.

It is worth mentioning that for $\mathrm{Chl} \mathbf{a}$, the zerocrossing of the $\triangle O D$ spectra remains constant (644 $\mathrm{nm}$ ) for all $\mathrm{S}_{1}$ populations from near-zero to $10 \%$. In the case of $20-\mathrm{Cl}-\mathrm{Chl}$ a, this wavelength is changed only minimally around $\lambda=654 \mathrm{~nm}(<3 \mathrm{~nm})$.

A fixed position of $\triangle O D=0$ irrespective of population changes is typical for two-level systems. At this wavelength, the excited-state absorption crosssection corresponds to the sum of the ground-state absorption cross-section and the cross-section of stimulated emission. This behaviour of chlorophyll solutions underlines the result of the numerical calculation, namely, that under the short-pulse illumination used, the triplet contribution to the $\triangle O D$ spectra is small (maximum value of relevant $T_{1}$ population is $10 \%$ of $S_{1}$ population for $20-\mathrm{Cl}-\mathrm{Chl}$ a, $5 \%$ of $S_{1}$ population for Chl a). Because of this small triplet contribution, an approximative $T_{1}$ spectrum in the spectral region of interest $(630-680 \mathrm{~nm})$, e.g. a constant value of $\sigma_{\mathrm{T}}=5 \times 10^{-17} \mathrm{~cm}^{2}$, was assumed in the above calculation of the $S_{1}$ absorptions crosssections from $\triangle O D$ spectra. This seems to be justified by the corresponding parameter identification in non-linear absorption (see above) and the triplet 

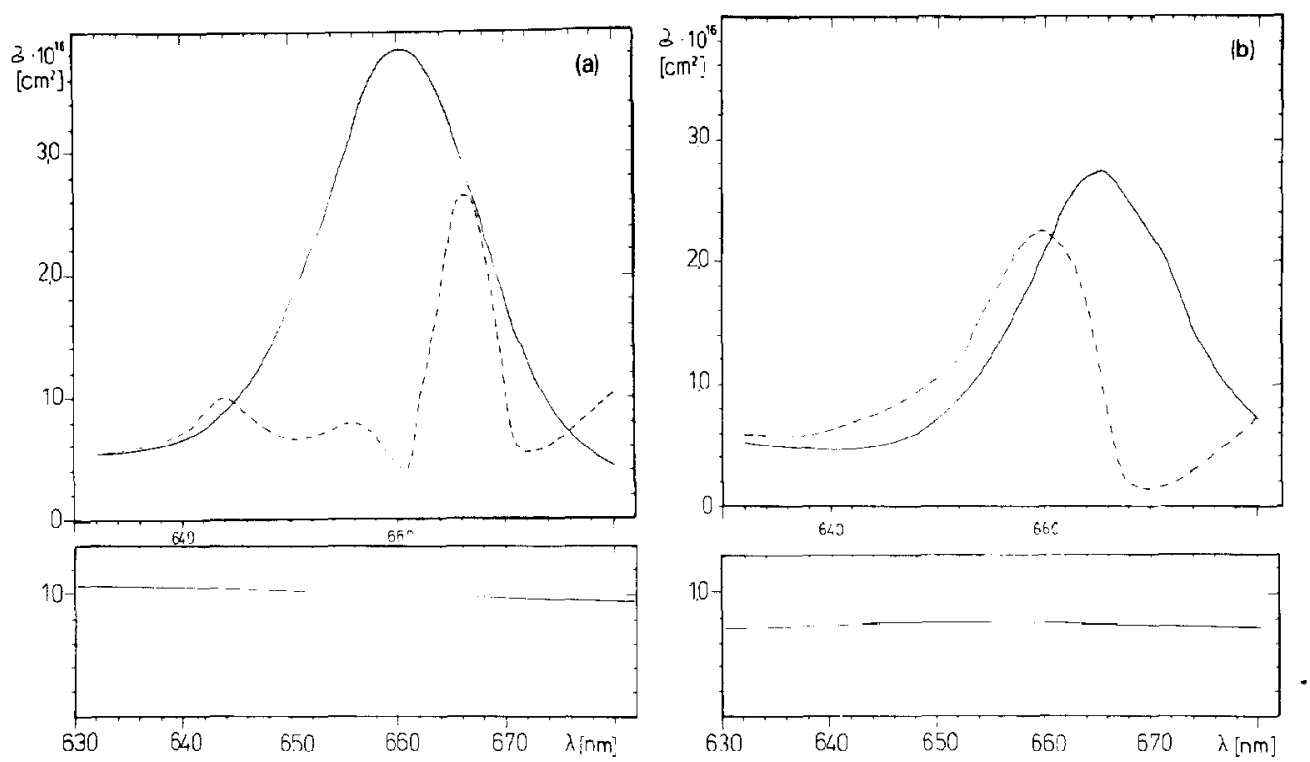

Fig. 3. Upper part: Absorption cross-sections from ground state (-) and first excited-singlet state (---) of $\mathrm{Chl}$ a (a) and 20-Cl-Chl a (b) in diethyl ether. Lower part: UV-ground-state absorption cross-sections of $\mathrm{Chl} \mathrm{a} \mathrm{(a)} \mathrm{and} \mathrm{20-Cl-Chl} \mathrm{a} \mathrm{(b)} \mathrm{in} \mathrm{ether,} \mathrm{but} \mathrm{the} \mathrm{wave-}$ lengths are transformed according to $\lambda=\left[\left(\lambda_{\mathbf{U V}}\right)^{-1}-\left(\lambda_{\mathrm{S}(0-0)-5(10)}\right)^{-1}\right]^{-1}$.

absorption data given in the literature $[21,22]$.

The $S_{1}$ absorption cross-sections for the ether solution of $\mathrm{Chl}$ a and $20-\mathrm{Cl}-\mathrm{Chl}$ a calculated this way are shown in fig. 3 . It should be noted that the stability of the calculated $S_{1}$ spectra around the zerocrossing wavelengths (vide supra) is a sensitive test of the set of excited-state constants used, because the $S_{1}$ cross-sections at these wavelengths can be calculated independently. For both samples, this test fits very well. Special attention should be drawn to the "hole" around the $670 \mathrm{~nm}$ in both spectra. With some scepticism, a similar hole was already seen by Shepanski and Anderson [23] in the $S_{1}$ absorption spectrum of Chl a in pyridine. This spectral feature is strongly supported by the lasing propertics of the chlorophyll solutions described below. It is noteworthy that with both pigments, there is no simple correspondence of the $S_{1}$ absorption spectra with the near-UV part of the $S_{0}$ absorption cross-sections, transformed into the visible region by subtracting the wavenumber of the respective $S_{0}-S_{1}(0-0)$ transition) (see also fig. 3).

\subsection{Lasing action}

Lasing properties are very informative with respect to first excited-electronic-state properties, especially with chlorophylls $[24,25]$. The results obtained in this study with the ether solutions for several different pumping conditions are shown in table 3.

The laser wavelengths are clearly dominated by the $S_{1}$ absorption profile: With restriction to two energy levels $\left(S_{0}\right.$ and $S_{1}$ with a continuum of sub-levels each) and without $S_{1}$ absorption, the threshold of self-tuned laser action would be expected at $679 \mathrm{~nm}$ for $\mathrm{Chl}$ a and at $682 \mathrm{~nm}$ for 20-Cl-Chl a (calculated from the data of fig. 1). By contrast, the experimental lasing wavelengths are much shorter: $671.5 \mathrm{~nm}$ for $\mathrm{Chl} \mathbf{a}$ and $675 \mathrm{~nm}$ for $20-\mathrm{Cl}-\mathrm{Chl} \mathrm{a}$; thus, they are located in the hole of the $S_{1}$ absorption. These results can be rationalized by the data given in fig. 3 and the above-mentioned assumption regarding $T_{1}$ absorption. This is shown in table 4 , where the calculated total gain in the lasing region is given for both samples. This calculation is based on population distributions following from the $\lambda$ derivation of the gain at threshuld. Table 4 shows, that the deviation of the 
Table 3

Lasing properties of $\mathrm{Chl}$ a and $20-\mathrm{Cl}-\mathrm{Chl} \mathrm{a}$ in ether solution ${ }^{\mathrm{a}}$ )

\begin{tabular}{|c|c|c|c|c|}
\hline \multicolumn{3}{|c|}{ Pump conditions } & \multirow{2}{*}{\multicolumn{2}{|c|}{$\begin{array}{l}\text { Laser threshold wave- } \\
\text { length }(\mathrm{nm})\end{array}$}} \\
\hline \multirow[b]{2}{*}{$\begin{array}{l}\lambda_{\text {pump }} \\
(\mathrm{nm})\end{array}$} & \multirow[b]{2}{*}{$\begin{array}{l}\text { power } \\
(k W)\end{array}$} & \multirow[b]{2}{*}{$\begin{array}{l}\text { duration } \\
\text { (ns) }\end{array}$} & & \\
\hline & & & $\mathrm{Chl} \mathrm{a}$ & $20-\mathrm{Cl}-\mathrm{Chl}$ a \\
\hline 337 (a) & 350 & 0.5 & - & - \\
\hline $660(a)$ & 60 & 0.5 & - & - \\
\hline 337 (a) & 250 & 1.7 & 671.5 & 675 \\
\hline 337 (a) & 800 & 7.0 & - & - \\
\hline 648 (b) & 150 & 6.0 & 669.5 & - \\
\hline
\end{tabular}

a) Concentrations: $10^{17}$ molecules $\mathrm{cm}^{-3}$ (corresponding to $5 \%$ transmission at $337 \mathrm{~nm}$ for $1 \mathrm{~mm}$ sample), transverse (a) and longitudinal (b) pump geometry, pump radiation focused by cylindrical (a) and spherical lens (b) $(f=5 \mathrm{~cm})$, non-dispersive resonator with $100 \%$ and $40 \%$ mirrors (a) and cuvette resonator (b), cavity length $4 \mathrm{~cm} \mathrm{(a)} \mathrm{and} 0.1 \mathrm{~cm} \mathrm{(b),} \mathrm{active}$ material length $1 \mathrm{~cm}$ (a).

calculated threshold wavelength from the experimental value is $\leqslant 1 \mathrm{~nm}$ for both solutions. A further confirmation of the calculated gain profile of the 20 $\mathrm{Cl}-\mathrm{Chl}$ a solution was obtained by extrinsic loss variation [24], e.g. for the broadest lasing bandwidth achieved, it holds that $G(672.3 \mathrm{~nm})=G(678 \mathrm{~nm})$, which agrees with the calculated profile. Because of the sensitive dependence of these calculated lasing properties on both the absolute values and $\lambda$ derivation of the $S_{1}$ absorption spectrum, the latter is well confirmed in this way. From the threshold consideration, it follows, also, that the lasing properties are sensitively influenced by the $S_{1}$ deactivation channels: Threshold can be reached only for the conditions $\left[S_{1}\right]>\left[S_{0}\right]$, and a certain triplet population is a prerequisite to laser action. This explains the negative results with the short-pulse pump regime (table
$3)$. On tc other hand, onc has to avoid dominating triplet populations. Pumping with the 6 ns dye laser pulse is an interesting intermediate case where threshold is reached with the $\mathrm{Chl}$ a solution, but not with the 20-Cl-Chl a solution, because of the nearly twofold ISC rate for the latter.

\section{Conclusions}

The excited state of 20-Cl-Chl a shows some pronounced differences as compared to the 20-unsubstituted pigment: (a) The $\mathrm{Cl}$ substituent leads to a red-shift of the $S_{0}-S_{1}$ absorption of circa $5 \mathrm{~nm}$; similar shifts are observed in other $\mathrm{C}$-20-substituted chlorophylls (see ref. [3]). The excited state shows, by contrast, a blue-shifted absorption, which indicates a structural change induced in the excited state by the $\mathrm{C}-20$ substituent. (b) The $\mathrm{Cl}$ substituent leads to a reduction of the fluorescence yield $[2,3]$. This is due to both increased radiationless deactivation, and intersystem crossing to the triplet state. (c) The increased photochemical reactivity is a further distinctive difference among the pigments. The differences are probably due to a combination of the steric hindrance introduced into the molecule by the substituent, of increased spin-orbit coupling originating from a heavy-atom effect, and possibly also of electronic factors induced by the electronegative substituent.

The results show that relatively minor changes in the molecular structures, which have only little effect on the ground-state properties, can influence the excited state of chlorophylls. Such changes can be of considerable importance in plants, which have to protect themselves from photodynamic damage in-

Table 4

Calculated gain/loss $\left(\mathrm{cm}^{-1}\right)$ for the ether solutions of 20-Cl-Chl a and $\mathrm{Chl} \mathbf{a}$, respectively, at the experimental conditions according to table 3, third row

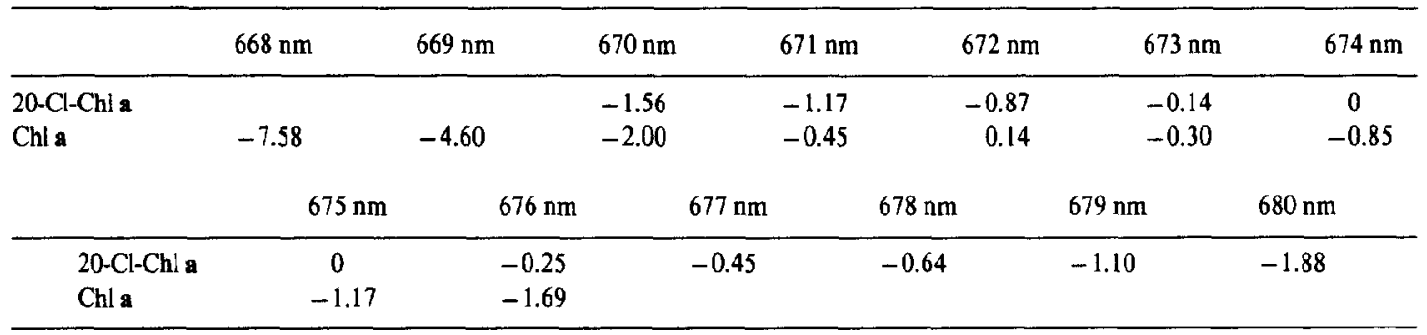


duced by functionally non-uncoupled chlorophylls, e.g. during biosynthesis and breakdown. They are also of interest with regard to the recent attempt to use chlorophyllous pigments in photodynamic therapy [26]. In both cases, a judicious choice of substituents can be an cssential factor for the physiological action.

\section{Acknowledgement}

AS and HS acknowledge support from the Deutsche Forschungsgemeinschaft (SFB 143, Project A1 ). AS is indebted to the Hanns-Seidel-Stiftung, Munich, for a stipend, and acknowledges a travel grant from the Deutscher Akademischer Austauschdienst and the Akademie der Wissenschaften der DDR. Part of this work was supported by the "Centre of Ultrafast Phenomena in Physics (Berlin/Jena)". We thank P. Hoffmann (Humboldt Universität, Berlin, GDR) for many helpful discussions.

\section{References}

[1] D. Dörnemann and H. Senger, FEBS Letters 126 (1981) 323

[2] D. Dörnemann and H. Senger, Photochem. Photobiol, 43 (1986) 573.

[3] H. Scheer, E. Gross, B. Nitsche, E. Cmiel, S. Schneider, W. Schäfer, H.-M. Schiebel and H.-R. Schulten, Photochem. Photobiol. 43 ( 1986 ) 559.

[4] M. Kobayashi, T. Watanabe, A. Struck, H. Scheer and M. Nakazato, FEBS Letters 235 (1988) 293.

[5] M. Senge, D. Dörnemann and H. Senger, FEBS Letters 234 (1988) 215

[6] M. Senge, A. Struck, D. Dörnemann, H. Scheer and H. Senger, $Z$, Naturforsch. $83 \mathrm{c}$ (1988) 515 .
[7] A. Struck, E. Cmiel, S. Schneider and H. Scheer, Photochem. Photobiol. 51 (1990) 217.

[8] D. Leupold, J. Ehlert, S. Oberländer and H. Stiel, in: Proceedings of the 3rd Symposium on Optical Spectroscopy, Reinhardsbrunn 1984, Teubner Texte zur Physik, Vol. 4 (Teubner, Leipzig, 1985) p. 111.

[9] D. Leupold, Dissertation B, Humboldt University of Berlin (1986).

[10] S. Dähne, D. Leupold and H. Stiel, Acta Phys. Polon. 5 (1987) 777 .

[11] D. Leupold, J. Ehlert, S. Oberländer, E. Klose, S. Mory and G. Winkelmann, Laser Chem. 10 (1989) 73.

[12] S. Oberländer and D. Leupold, Opt. Commun. 52 (1984) 57.

[13] D. Leupold, M. Scholz and J. Ehlert, Chem. Phys. Letters 115 (1985) 434.

[14] M. Jabben, N.A. Garcia, S.E. Braslavsky and K. Schaffner, Photochem. Photobiol. 43 (1986) 127.

[15] II. Lucht, K. Biehler, W. Nebe and K.-E. Süsse, Jenaer Rundschau 30 (1985) 170.

[16] H. Stiel, K. Teuchner and W. Becker, Exp. Technik Phys., submitled for publication.

[17] O.G. Peterson, J.P. Webb and W.C. Mc Colgin, J. Appl. Phys. 42 (1971) 1917

[18] H.K. Lichtenthaler, Methods in enzymology, Vol. 148 (Academic Press, New York, 1987) p. 362.

[19] S. Drexhage, in: Dye lasers, ed. F.P. Schäfer (Springer, Berlin, 1973).

[20] D. Leupold and J. Ehlert, Verfahren zur optischen Präparation hochangeregter reaktiver molekularer Spezies, GDR patent WPG OIN $\backslash 3119482$.

[21] H. Linschitz and K. Sarkanen, J. Am. Chem. Soc. 80 (1958) 4826

[22] D. Leupold, H. Stiel and J. Sepiof, Chem. Phys. Letters 132 (1986) 137

[23] J.F. Shepanski and R.W.J. Anderson, Chem. Phys. Letters 78 (1981) 165 .

[24] D. Leupold, S. Mory, R. Konig, P. Hoffmann and B. Hieke, Chem. Phys. Letters 45 (1977) 567.

[25] J.C. Hindman, R. Kugel, A. Svirmickas and J.J. Katz, Proc Natl. Acad. Sci. US 74 (1977) 5.

[26] J.D. Spikes and J.C. Bommer, in: The chlorophylls, ed. H Scheer (CRC Press, Boca Raton), in press. 